\title{
An Economic Lighting Management System of the Internet of Things for Smart Home Application
}

\author{
Chao-Yang Lee ${ }^{1, a}$, Chu-Sing Yang ${ }^{2, b}$ \\ ${ }^{1}$ Institute of Computer and Communication Engineering, Department of Electrical Engineering, \\ National Cheng Kung University, Tainan, Taiwan, R.O.C \\ aemail: nelsorn@gmail.com, bemail: csyang@ee.ncku.edu.tw
}

Keywords: Economic Lighting Management; Internet of Things; Smart Home Application

\begin{abstract}
The energy issues which is in the internet of thing technology for smart home application is an important problem. Due to the sunlight, energy consumption of lighting cause the unnecessary power utility. This study proposed an economic lighting management system for smart home application. The economic lighting management strategy is based on internet of thing technology. All devices which are include cameras, lighting control units, and perception device are connection via Ethernet or Bluetooth. The experiment results show that economic lighting management system can carry out well in a smart home.
\end{abstract}

\section{Introduction}

Recently, smart home technology continues to be a very popular technology to energy utilizing more efficiently than traditional buildings [1]. Several studies [2] had proposed to build smart home for reducing energy consumption. Mingfu Li and Hung-Ju Lin [3] proposed a smart home control system based on wireless sensor networks and power line communications. This study armed to reduce the unnecessary energy consumption of a smart home by using the smart control algorithm to reduce the impact of wireless interference on a smart home control network. The smart control algorithm can be reduced by at least $40 \%$ in smart home. Hemant Ghayvat et al. [4] proposed a smart home system based on wireless sensor network (WSN) protocols to address the smart homes for assisted living. The protocol of smart home system, which is Wellness Sensor Networks, had developed for ambient assisted living and provided a safe environment.

This study focuses on to solve the lighting management issue in smart home. The sunlight in a room had shown in Fig. 1. The lighting near the window is lightness, and the lighting near the door is twilight. Normally, the human wants to light, when he lives in a twilight area. Thus, the human open the lamp, as shown in Fig. 2. However, the lighting near the window is unnecessary, because the sunlight is enough for lighting. The economic lighting management system arm to reduce the unnecessary lighting for illumination and promotes the energy consumption more efficiently.

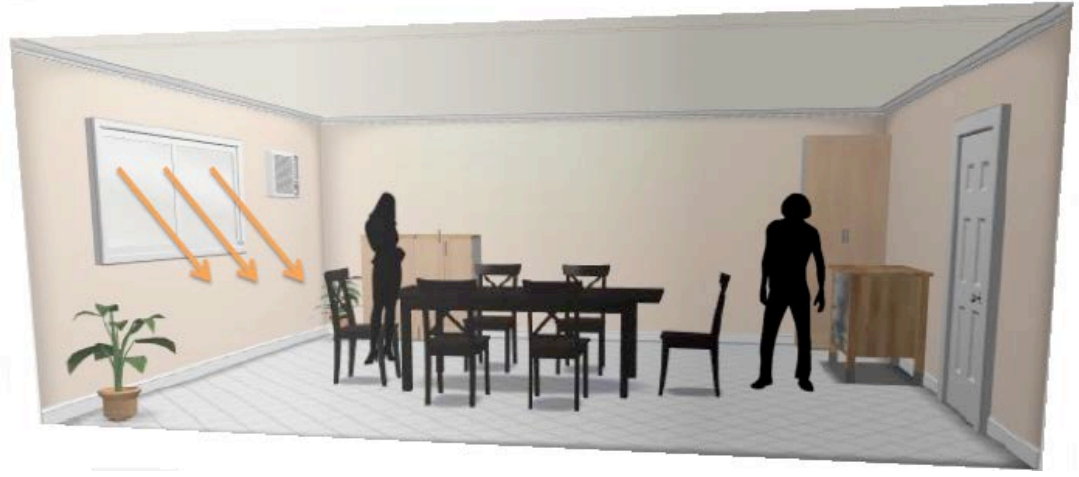

Fig.1. The sunlight in a room 


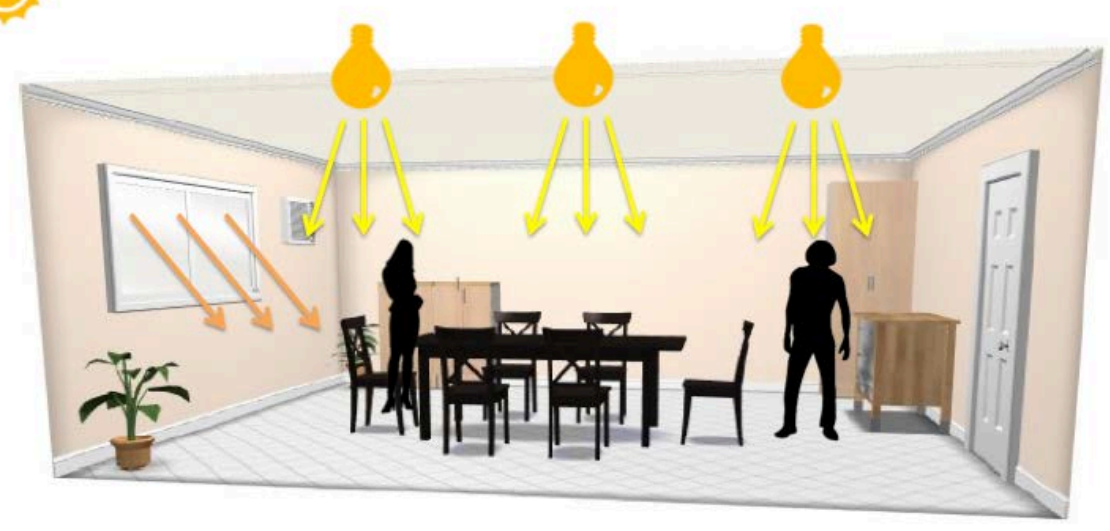

Fig.2. The illumination in a room

\section{Design of the Economic Lighting Management System}

This study proposed a smart economy strategy which called economic lighting management system (ELMS) for smart home application. The ELMS is based on internet of thing technology. All devices in the smart home field are connecting with each other through Ethernet or Bluetooth and construct a network of internet of things. The Fig. 1 shows the architecture of ELMS. It includes visual sensing model and management model. The visual sensing model has 2 devices which are a lot of cameras and a perception device. The cameras arm to obtain the patterns of real work environment in a room, and transmit the patterns to perception device via Ethernet. An indoor room need built least one camera for sensing. The perception device analyzes the intensity of real work environment from patterns in a room. All intensity information sends to economic lighting management model via Ethernet. The economic lighting management model carry out the economic lighting management strategy and control the brightness of all lighting via pulse density modulation (PDM) technology. The light connection by using Bluetooth and all lights construct a network.

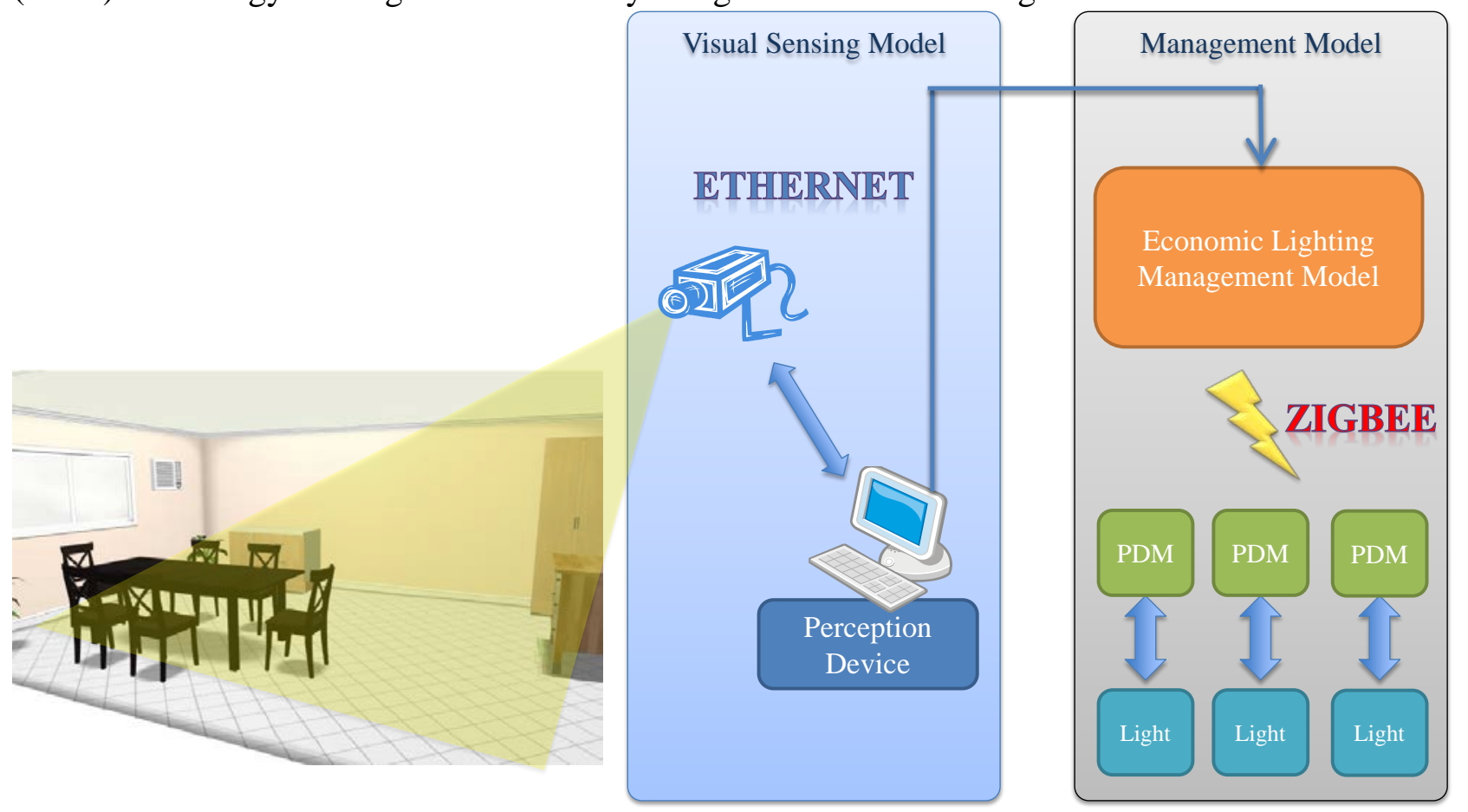

Fig.3. The architecture of ELMS 


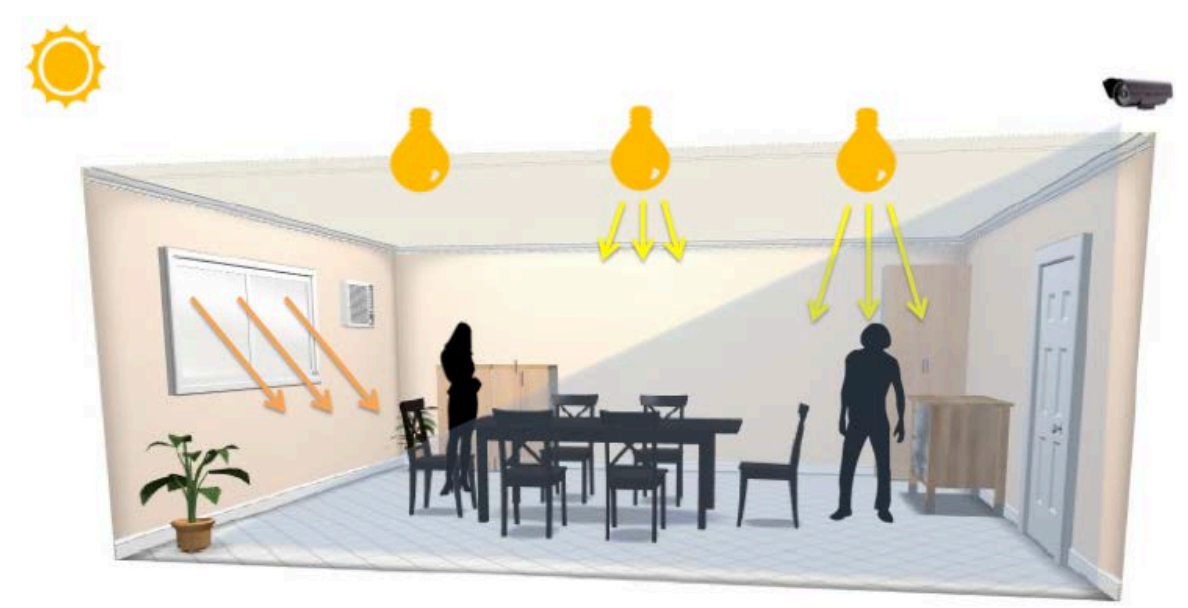

Fig.4. The economic lighting management strategy

Fig. 4 shows the economic lighting management strategy for smart home. This study employs the camera to sensing the environment in a room. The camera captures the images and sends it to perception device for illumination analysis. The illumination near the windows has the lighting sufficiently than the area near the door. Thus, the lamp near the window is close. The illumination near the door has the lighting insufficiently than the area near the window. Therefore, the lamp near the door is lighting in $100 \%$ effectiveness. The lamp on the door is lighting in $50 \%$ effectiveness. These three lighting control can provide the adequate illumination whole room.

\section{Experiment results}

The experiment environment used 8 LED lighting and an AXIS M1054 camera on ceiling. Fig. 5 shows the experiment area. The lighting control via the economic lighting management system called VA+CA method. The lighting control only control the lighting near the window called VA method. The tradition method is non-control for lighting. Fig. 6 shows the experiment results. The proposed economic lighting management system can reduce the unnecessary the energy consumption of lighting for power saving in smart home.

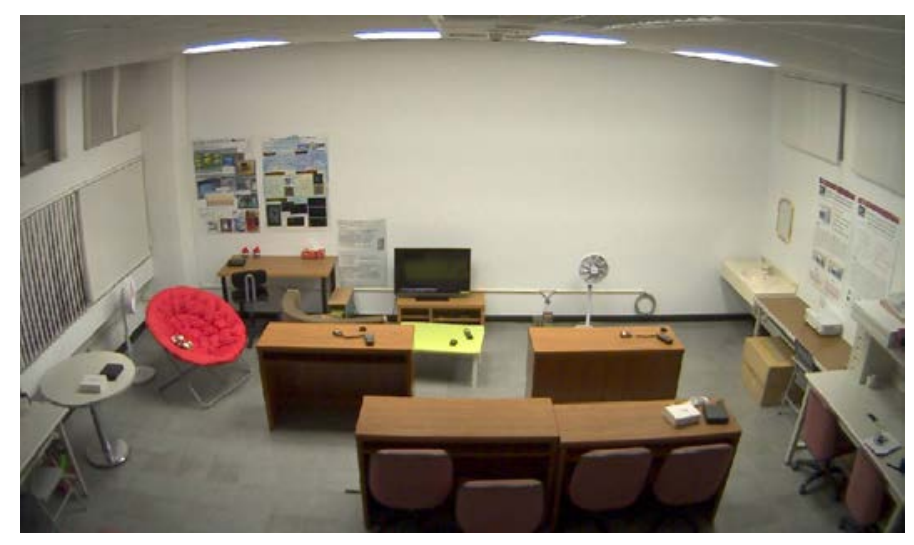

Fig.5. The experimental area 


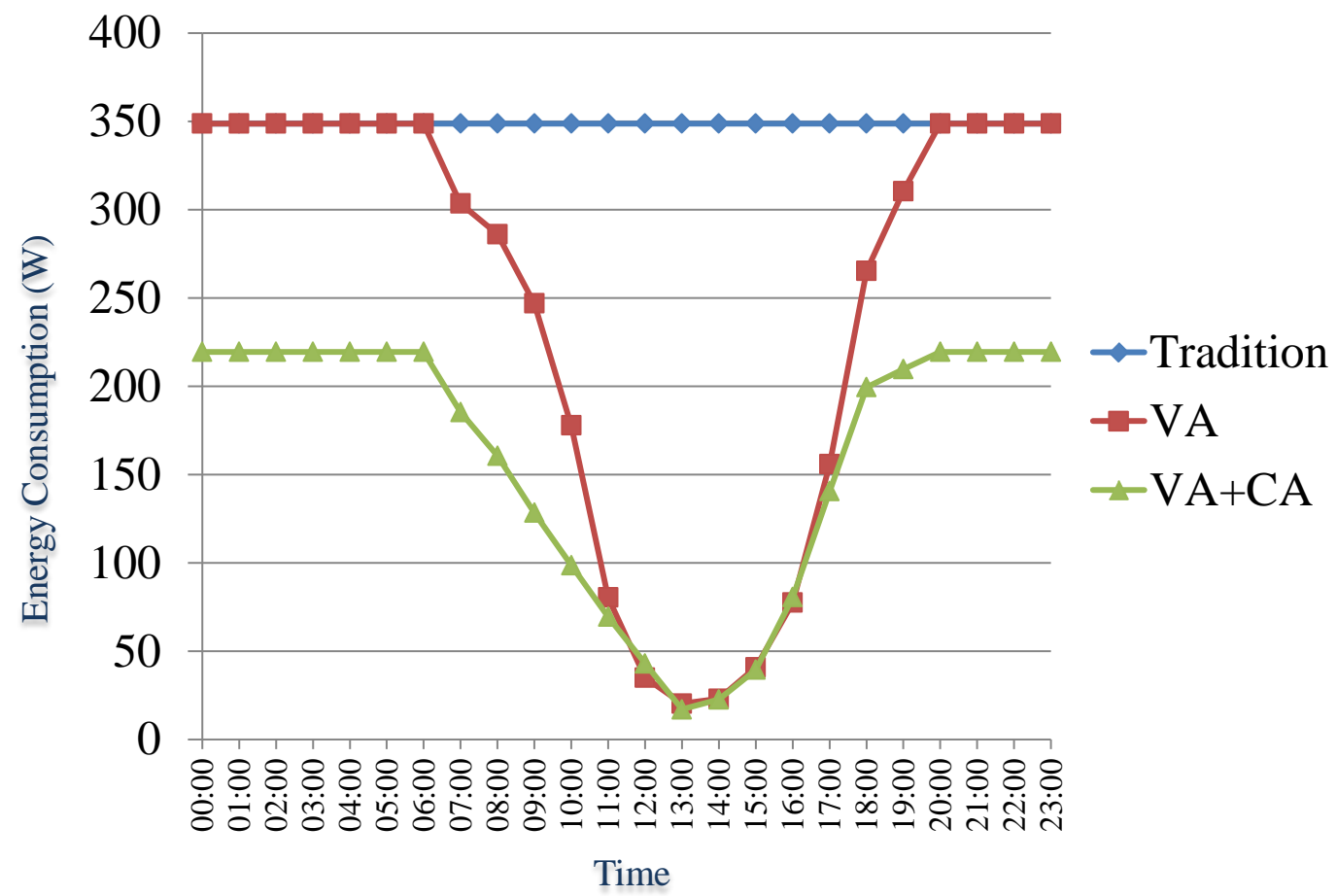

Fig.6. The experimental results

\section{Conclusion}

This study proposed a smart economy strategy which called economic lighting management system (ELMS) for smart home application. The ELMS is based on internet of thing technology. All devices in the smart home field are connecting with each other through Ethernet or Bluetooth and construct a network of internet of things. The experiment environment used 8 LED lighting and an AXIS M1054 camera on ceiling. The proposed economic lighting management system can reduce the unnecessary the energy consumption of lighting for power saving in smart home.

\section{Acknowledgment}

This work is supported in part by National Science Council under grant MOST 104-2221-E-006 -119 -MY3, Taiwan.

\section{References}

[1] Chao-Lin Wu, Li-Chen Fu. Design and Realization of a Framework for Human-System Interaction in Smart Homes. IEEE Transactions on Systems, Man, and Cybernetics. 201242 (1) 15-31.

[2] Dae-Man Han, Jae-Hyun Lim. Smart Home Energy Management System using IEEE 802.15.4 and ZigBee. IEEE Transactions on Consumer Electronics. 201056 (3) 1403 - 1410.

[3] Mingfu Li, Hung-Ju Lin. Design and Implementation of Smart Home Control Systems Based on Wireless Sensor Networks and Power Line Communications. IEEE Transactions on Industrial Electronics, 201562 (7) 4330-4442.

[4] Hemant Ghayvat, Jie Liu, Subhas Chandra Mukhopadhyay, Xiang Gui. Wellness Sensor Networks: A Proposal and Implementation for Smart Home for Assisted Living. IEEE Sensors Journal. 201515 (12) 7341 - 7348. 
[5] Y. Zhang, R. Yu, S. Xie, W. Yao, Y. Xiao, and M. Guizani, "Home M2M Networks: Architectures, Standards, and QoS Improvement," IEEE Communications Magazine, Vol. 49, Issue 4, pp. 44-52, 2011. 